\title{
Inteligencia emocional y estilos educativos en docentes de instituciones educativas privadas religiosas 2015, Chosica
}

\section{Emotional intelligence and educational styles in teachers of Private and Religious Educational Institutions 2015, Chosica}

\author{
Segura Yauri, Alessandra Stelly ; Quinteros Zúñiga, Dámaris Susana \\ EP Psicología, Universidad Peruana Unión
}

\begin{abstract}
Resumen
La presente investigación tuvo como objetivo identificar la relación entre la inteligencia emocional y los estilos educativos en docentes. Se desarrolló un diseño no experimental de corte transversal de alcance descriptivo correlacional. La muestra estuvo compuesta por 77 docentes de ambos géneros que, adscritos a seis instituciones educativas, privadas y religiosas de Chosica, Lima, Perú. Los instrumentos empleados fueron el Inventario de Inteligencia Emocional (ICE), de Baron; y el Perfil de Estilos Educativos, de Magaz y García. Según el análisis de correlación, el coeficiente de Pearson indicó que, si existe relación significativa e inversa entre las variables, indicando que, a mayor nivel de inteligencia emocional en el docente, menores fueron los puntajes en los estilos educativos sobreprotector y punitivo. Sin embargo, el estilo educativo asertivo e inhibicionista, serían independientes del nivel de inteligencia emocional del docente.
\end{abstract}

Palabras clave: inteligencia emocional, estilos educativos, docentes, psicología educativa.

\begin{abstract}
The present research aimed to identify the relationship between emotional intelligence and educational styles in teachers. A non-experimental cross-sectional design with descriptive correlation was developed. The sample consisted of 77 teachers of both sexes who were assigned to six educational, private and religious institutions of Chosica, Lima, Peru. The instruments used were Baron's Inventory of Emotional Intelligence (ICE); And the Profile of Educational Styles, by Magaz and García. According to the correlation analysis, the Pearson coefficient indicated that if there is a significant and inverse relationship between the variables, indicating that the higher the level of emotional intelligence in the teacher, the lower the scores on the overprotective and punitive educational styles. However, the assertive and inhibitionist educational style, would be independent of the level of emotional intelligence of the teacher.
\end{abstract}

Key words: emotional intelligence, educational styles, teachers, educational psychology.

\section{Introducción}

El ejercicio profesional de un educador, es un trabajo basado en las interacciones sociales. En este sentido, la docencia es una de las profesiones más estresantes, ya que requiere un serio esfuerzo para regular no sólo las propias emociones, sino también, las de los estudiantes, padres y compañeros de trabajo (Fernández, 2005). Así el manejo emocional del docente de sus actitudes y reacciones ante situaciones diversas, es bastante significativa sobre los estudiantes (López-Goñi y Goñi, 2009).

El docente necesita desarrollar competencias emocionales por dos razones contundentes. Primero, porque en las aulas, los estudiantes se llevan el mayor impacto de modelo socio- emocional adulto; y segundo, porque adecuados niveles de inteligencia emocional ayudan al docente a afrontar con mayor éxito el estrés laboral en el contexto educativo (Extremera y Fernández-Berrocal, 2004).

En este sentido, el proceso de educación no sólo consiste en la transmisión de conocimientos, sino también en la transmisión de patrones de conducta, ya que el docente en el aula se convierte en un modelo de aprendizaje vicario a través del cual el estudiante aprende a expresar, regular y pensar todas esas pequeñas frustraciones que transcurren durante el proceso de aprendizaje en el aula. Por lo que se podría afirmar que la falta de inteligencia emocional en el docente afecta directamente el proceso de aprendizaje (Extremera y Fernández-Berrocal, 2004). 
Sala (2002) refiere que la inteligencia emocional influye de manera significativa en los estilos educativos, explica que los estudiantes de docencia que obtuvieron puntuaciones positivas en la competencia de estado afectivo, también tuvieron altas puntuaciones en el estilo educativo asertivo.

Por otro lado, se define como "estilo" al conjunto los rasgos tanto de personalidad, como cognitivos, afectivos y fisiológicos, que sirven de indicadores sobre cómo el ser humano percibe, procesa la información, forma conceptos, reacciona y se comporta (Hervás, citado por Freitas, 2012). Según Magáz y García (1998) los estilos educativos vienen a ser el conjunto de ideas, creencias, valores, actitudes y hábitos de comportamiento que especialmente padres y docentes mantienen respecto a la educación de los hijos y estudiantes. Los mismos autores afirman además que todas las personas, desde la infancia hasta la madurez, al estar inmersas en un medio social, son influenciadas por el entorno, tanto a través de modelos directos como padres, hermanos, tíos, abuelos y profesores, y de modelos indirectos como la televisión, prensa, radio, etc. A su vez este proceso facilita la adquisición de diversas ideas, creencias y valores que configuran la percepción del mundo. Sumado a esto están las propias experiencias que modulan lo que finalmente constituirían los hábitos comportamentales, las formas de interacción con los demás y con uno mismo. No sólo es necesario que el docente sea emocionalmente inteligente para emitir un modelo adecuado a los estudiantes, sino que además debe ser capaz de establecer una relación emocional con éstos con el objetivo de formar el carácter lo suficientemente estable, para que el estudiante no sólo posea conocimientos, sino que además tenga la habilidad de hacerle frente a la vida y tomar decisiones que tengan una repercusión positiva a nivel personal y colectivo (Cerrillo, 2003).

El docente, en el aula de clase es el gestor, éste es quien promueve el clima que el aula tendrá durante su estadía. Todo lo que el docente realice, será observado por los estudiantes, de ahí que su papel en el aula es protagónico, porque dependerá del docente el cómo se construyan las relaciones con los estudiantes y de éstos con otros estudiantes dentro de la clase. Es por ello que se enfatiza en la importancia de que el docente tenga claro lo que quiere expresar y por consecuencia, expresarlo de manera correcta (Barreda, 2012).

Villán (2013) en España, investigó sobre los estilos de enseñanza y su relación con las competencias emocionales en docentes de educación infantil, en una muestra de 121 docentes de diversas comunidades autónomas. Se encontró una fuerte relación de la inteligencia emocional con la dimensión socioemocional deduciendo que la inteligencia emocional de los maestros va a ser influyente en el ejercicio docente con sus alumnos, en aspectos como empatía, mediación y sensibilidad comunicativa. Así también encontró una relación considerable entre la inteligencia emocional y la dimensión comunicativa-relacional, lo cual refiere que el nivel de la inteligencia emocional desarrollado por el docente es importante e influyente en aspectos como asertividad, resolución de conflictos y liderazgo afectivo del ejercicio docente en el aula de clases. Lo que lleva a la afirmación de la autora que el nivel de competencia emocional tiene influencia en los estilos de enseñanza del docente, sin embargo, no encontró índices altos de predicción (35.5\%), a pesar de ello considera importante tener en cuenta ésta información para mejorar la práctica docente.

Huanca (2012), investigó los niveles de inteligencia emocional en los docentes debido a la problemática actual sobre la enseñanza, ya que es muy mencionado en este ámbito que la capacidad emocional del docente potenciará el trabajo pedagógico en los estudiantes, formándolos no solo en el aspecto académico sino también en el aspecto emocional. Las investigaciones en cuanto a la inteligencia emocional en el ámbito educativo se han incrementado, ya que ésta favorece el clima escolar y por consiguiente el proceso pedagógico. La muestra estuvo conformada por docentes de una institución educativa del distrito de Ventanilla - Callao en Lima, Perú, en 60 docentes de ambos sexos, a los cuales se les administró el Inventario de Inteligencia Emocional de Baron; encontrando que los docentes que manejan de manera adecuada los componentes de la inteligencia emocional evidencian un adecuado ajuste psicológico y bienestar emocional, presentando mayor calidad y cantidad de redes interpersonales y de apoyo social; asimismo, son menos propensos a realizar comportamientos agresivos o violentos, logran obtener mayor rendimiento escolar de sus estudiantes al enfrentarse a situaciones de estrés con mayor facilidad. Por lo mencionado, el objetivo del estudio fue determinar si existe relación significativa entre inteligencia emocional y estilos educativos en docentes de instituciones educativas privadas religiosas de Chosica, 2015.

\section{Método}

La investigación tiene un alcance correlacional-descriptivo, ya que se especifican las características encontradas en la relación existente entre las variables de estudio, además el diseño de la investigación siguió una orientación no experimental de corte transversal, ya que no se manipularon las variables y los datos fueron tomados en un tiempo único (Hernández, Fernández y Baptista; 2010). El estudio se desarrolló en el distrito de Lurigancho - Chosica, ubicado en el departamento de Lima, Perú.

El grupo de participantes estuvo conformado por 77 docentes activos de Instituciones Privadas Religiosas de Chosica, de ambos sexos y con edades entre 21 a 60 años. El tipo de muestreo realizado fue no probabilístico, a conveniencia del investigador. Para la evaluación de la inteligencia emocional se utilizó el Inventario de Inteligencia Emocional de Baron (I-CE), el cual consta de 133 afirmaciones que el examinado responde según una escala tipo Likert con cinco posibilidades, que van desde "casi nunca" a "siempre". Dicho cuestionario ha sido validado por Ugarriza (2003) con baremos peruanos. El cuestionario pretende valorar los componentes de la inteligencia emocional los cuales son cinco: intrapersonal, interpersonal, adaptabilidad, estado afectivo y gestión del estrés. La confiabilidad del instrumento es de 0.93, apreciándose una consistencia interna muy alta. Así también se observa que la validez es significativa según la prueba de chi cuadrado, con las siguientes valoraciones; para la inteligencia intrapersonal 0.92, 
interpersonal 0.68, inteligencia de adaptabilidad 0.78, inteligencia manejo del estrés 0.61 , inteligencia ánimo general 0.88 (Ugarriza, 2001).

En el presente estudio se analizaron las propiedades psicométricas de este inventario, encontrando un alfa de Cronbach de 0.928, lo cual evidencia adecuada fiabilidad de la prueba. De igual manera se obtuvo un alfa de 0.876 en la dimensión intrapersonal, 0.748 en la dimensión interpersonal, 0.807 en la dimensión de adaptabilidad, 0.849 en el manejo del estrés y 0.753 en la dimensión de ánimo general. Los puntos de corte establecidos y utilizados en esta investigación están consignados en la Hoja de Perfil de I-CE cuyos valores son los siguientes: (a) 116 a más, indica capacidad emocional muy desarrollada, (b) 85 a 115, indica capacidad emocional adecuada y (c) 84 y menos, indica capacidad emocional por mejorar.

Para la medición de los estilos educativos, se aplicó el instrumento Perfil de Estilos Educativos (PEE), elaborado y validado por Magaz y García (1998) con un alpha de Cronbach de 0.79, que indica que el instrumento cuenta con una adecuada fiablidad. Consta de 48 afirmaciones con alternativa de respuesta dicotómica. Este valora cualitativa y cuantitativamente las actitudes y valores ante la educación de los alumnos e hijos, así también permite cuantificar las ideas, actitudes y valores correspondientes a los estilos sobreprotector, punitivo, inhibicionista y asertivo. Para fines de la presente investigación también se analizó la validez de este test mediante el coeficientede validación V de Aiken, encontrándose una valoración de .92, lo cual indica adecuada validez. Y el alfa de Cronbach alcanzó un valor de .55, indicando adecuada fiabilidad global del test.

Para realizar la recolección de datos, se envió la carta de solicitud a los directores de las respectivas instituciones educativas, quienes asignaron la fecha, hora y lugar de evaluación. Las evaluaciones se realizaron en los ambientes de cada institución. Una vez reunidos los docentes, recibieron información breve acerca de las variables de estudio y el propósito de la investigación. Inmediatamente recibieron los test con sus respectivas instrucciones, se absolvieron algunas dudas pertinentes al desarrollo de los cuestionarios. Cada grupo tardó entre 40 a 60 minutos en completar ambos cuestionarios. Finalmente, cada docente recibió un presente en agradecimiento por su participación voluntaria. Para procesar los datos recolectados en la muestra, se introdujo todo lo obtenido en el programa estadístico SPSS versión 20. Tras la codificación de los datos, se transfirieron a una matriz para ser depurados y proceder al análisis. Los análisis iniciales fueron descriptivos con el objetivo de fijar de un modo más concreto las características de la muestra. Luego se realizó el análisis correlación con el coeficiente de Spearman, a fin de responder a los objetivos e hipótesis planteados.

\section{Resultados y discusión}

En la tabla 1 se observa que el 54.5\% de docentes evaluados presentaron un nivel muy desarrollado de inteligencia emocional, y el $45.5 \%$ restante se ubicó en un nivel adecuado. Respecto a la inteligencia intrapersonal el mayor porcentaje de docentes se encontró en un nivel adecuado (77.9\%). En relación a la inteligencia interpersonal, la mayoría de docentes obtuvo un nivel muy desarrollado (81.8). En adaptabilidad el $62.3 \%$ de docentes evaluados obtuvo un nivel adecuado. En cuanto al manejo del estrés el mayor porcentaje de docentes $(67.5 \%)$ se ubicó en el nivel adecuado. Finalmente, en cuanto al ánimo general también se observó al mayor porcentaje de docente con un nivel adecuado $(81.8 \%)$.

Tabla 1

Niveles de Inteligencia Emocional de los docentes de Instituciones Educativas Privadas Religiosas 2015, Chosica.

\begin{tabular}{lcccccc}
\hline & \multicolumn{4}{c}{ Nivel de desarrollo } & & \\
Inteligencia & Por mejorar & & Adecuado & Muy desarrollado \\
\cline { 2 - 7 } emocional & $\mathrm{f}$ & $\%$ & $\mathrm{f}$ & $\%$ & $\mathrm{f}$ & $\%$ \\
\hline Global & - & - & 35 & $45.5 \%$ & 42 & $54.5 \%$ \\
Intrapersonal & 8 & $10.4 \%$ & 60 & $77.9 \%$ & 9 & $11.7 \%$ \\
Interpersonal & - & - & 14 & $18.2 \%$ & 63 & $81.8 \%$ \\
Adaptabilidad & 5 & $6.5 \%$ & 48 & $62.3 \%$ & 24 & $31.2 \%$ \\
Manejo del estrés & 2 & $2.6 \%$ & 52 & $67.5 \%$ & 23 & $29.9 \%$ \\
Ánimo general & 2 & $2.6 \%$ & 63 & $81.8 \%$ & 12 & $15.6 \%$ \\
\hline
\end{tabular}

En cuanto a los estilos educativos de los docentes se observan los siguientes resultados: Se observa en la Tabla 2 que el $90,9 \%$ de docentes obtuvieron altas puntuaciones en el estilo asertivo, el 51,9\% obtuvo puntuaciones moderadas en el estilo inhibicionista, en el estilo sobreprotector se obtuvo un mayor porcentaje en el nivel moderado con 59,7\% y en el estilo punitivo se obtuvo un $24,7 \%$ también en nivel moderado. En cuanto a las puntuaciones bajas se observó que, en el estilo asertivo, los docentes no obtuvieron bajas puntuaciones, mientras que en el estilo punitivo se observa un $74 \%$, en el estilo inhibicionista se obtuvo un $40,3 \%$ y en el estilo sobreprotector se encontró un $23,4 \%$. 
Tabla 2

Puntuaciones de Estilos Educativos de los docentes de Instituciones Educativas Privadas Religiosas 2015, Chosica.

\begin{tabular}{lrccccc}
\hline & \multicolumn{7}{c}{ Puntuaciones en estilos educativos } \\
\cline { 2 - 7 } Estilos & \multicolumn{2}{c}{ Bajo } & \multicolumn{2}{c}{ Moderado } & \multicolumn{2}{c}{ Alto } \\
educativos & $\mathrm{f}$ & $\%$ & $\mathrm{f}$ & $\%$ & $\mathrm{f}$ & $\%$ \\
\hline Sobreprotector & 18 & $23,4 \%$ & 46 & $59,7 \%$ & 13 & $16,9 \%$ \\
Asertivo & - & - & 7 & $9,1 \%$ & 70 & $90,9 \%$ \\
Punitivo & 57 & $74,0 \%$ & 19 & $24,7 \%$ & 1 & $1,3 \%$ \\
Inhibicionista & 31 & $40,3 \%$ & 40 & $51,9 \%$ & 6 & $7,8 \%$ \\
\hline
\end{tabular}

Respecto a la relación entre las variables de estudio, se observó lo siguiente: En la tabla 3 se observa una relación estadísticamente significativa, moderada e indirecta entre el estilo de sobreprotección y la inteligencia emocional global (rs=-0.401, $\mathrm{p}<0.01)$; asimismo, con la inteligencia intrapersonal ( $\mathrm{rs}=-0.401, \mathrm{p}<0.01)$, con la interpersonal $(\mathrm{rs}=-0.320, \mathrm{p}<0.01)$; con la adaptabilidad ( $\mathrm{rs}=-0.420, \mathrm{p}<0.01$ ); con el manejo del estrés ( $\mathrm{rs}=-0.243, \mathrm{p}<0.05$ ); y con el ánimo general ( $\mathrm{rs}=-0.324$, $\mathrm{p}<0.01$ ). También se halló relación moderada e indirecta entre el estilo de punición y la inteligencia emocional (rs=-0.511, p<0.01), de igual manera con la inteligencia intrapersonal ( $\mathrm{rs}=-0.426, \mathrm{p}<0.01)$, con la interpersonal $(\mathrm{rs}=-0.358, \mathrm{p}<0.01)$, con la adaptabilidad $(\mathrm{rs}=-$ $0.441, \mathrm{p}<0.01)$, con el manejo del estrés ( $\mathrm{rs}=-0.398, \mathrm{p}<0.01)$ y con ánimo general $(\mathrm{rs}=-0.468, \mathrm{p}<0.01)$.

Tabla 3

Prueba de correlación entre estilos educativos e inteligencia emocional y dimensiones según la prueba Rho de Spearman

\begin{tabular}{lccccccccc}
\hline \multirow{2}{*}{ Dimensiones } & \multicolumn{2}{c}{ Sobreprotector } & \multicolumn{2}{c}{ Asertivo } & \multicolumn{2}{c}{ Punitivo } & \multicolumn{2}{c}{ Inhibicionista } \\
\cline { 2 - 9 } & rho & $\mathrm{p}$ & rho & $\mathrm{p}$ & rho & $\mathrm{p}$ & rho & $\mathrm{p}$ \\
\hline Inteligencia & $-.401^{* *}$ & 0.000 & .116 & 0.314 & $-.511^{* *}$ & 0.000 & -.028 & 0.809 \\
emocional & $-.401^{* *}$ & 0.000 & .154 & 0.180 & $-.426^{* *}$ & 0.000 & -.056 & 0.631 \\
Intrapersonal & $-.320^{* *}$ & 0.005 & .041 & 0.723 & $-.358^{* *}$ & 0.001 & .016 & 0.892 \\
Interpersonal & $-.420^{* *}$ & 0.000 & .140 & 0.223 & $-.441^{* *}$ & 0.000 & .057 & 0.620 \\
Adaptabilidad & $-.243^{*}$ & 0.033 & .115 & 0.318 & $-.398^{* *}$ & 0.000 & -.093 & 0.420 \\
Manejo del & $-.324^{* *}$ & 0.004 & .111 & 0.338 & $-.468^{* *}$ & 0.000 & -.017 & 0.881 \\
estrés & & & & & & & & \\
Ánimo general & & & & & & & & &
\end{tabular}

\section{Discusión}

Tras los resultados encontrados se observó que existe una relación inversa entre la inteligencia emocional y los estilos educativos sobreprotector y punitivo. Es así que los niveles de inteligencia emocional son menores cuando se obtiene un estilo educativo sobreprotector o punitivo. Este resultado encuentra asidero en la revisión bibliográfica, ya que según Baron (citado por Ugarriza, 2001), la inteligencia emocional en niveles adecuados o muy desarrollados, se vincular al comportamiento equilibrado, capaz de afrontar las demandas del entorno. De manera similar Extremera, Fernández-Berrocal y Durán (2003) concluyeron, tras encontrar que los docentes que poseen ciertas habilidades emocionales pueden hacerles frente a niveles intensos de estrés y ansiedad y evitar desajustes emocionales serios; lo que no se observaría en un docente con estilo sobreprotector. Según Magaz y García (1998), el estilo sobreprotector evidencia un fuerte e intenso sentimiento de responsabilidad, así como la culpabilización frente a los errores que sus estudiantes pudieran cometer. En esta línea de pensamiento, Villán (2013), encontró que docentes con inteligencia emocional desarrollada, evidencian mejor interacción con sus estudiantes como liderazgo afectivo y resolución de conflictos. En el caso del estilo punitivo, suele caracterizarse por un alto nivel de exigencia, intolerancia e incomprensión hacia sus estudiantes, lo cual está en contraste con niveles adecuados o muy desarrollados de inteligencia emocional.

Saarni (citado por Bisquerra, 2003), hace referencia que la competencia emocional se relaciona con la demostración de autoeficacia al expresar emociones en las transacciones sociales, sin embargo, ésta autoeficacia no se observaría en los estilos educativos sobreprotector y punitivo. Asimismo, Extremera y Fernández-Berrocal (2004), señalan que cuando el docente muestra mayores competencias emocionales desarrolladas, su estilo educativo será más asertivo, afectivo y estable. En cuanto al estilo asertivo e inhibicionista no se observa relación significativa y como refiere Sala (2002), esto puede deberse a que la influencia de la inteligencia emocional en los estilos educativos es moderada y compleja y cabe la probabilidad de que incidan otros factores. 
En este estudio también se halló relación significativa inversa entre la dimensión intrapersonal y los estilos educativos sobreprotector y punitivo. La inteligencia intrapersonal hace referencia a habilidades tales como percatarse y comprender los propios sentimientos, diferenciarlos y conocer su origen, así también la habilidad para expresar asertivamente sentimientos, creencias y pensamientos; involucra además la comprensión, aceptación y respeto por uno mismo conociendo las propias posibilidades y limitaciones; la capacidad de autorrealización e independencia también son habilidades consideradas en este componente, que como es claro, difícilmente aparecen caracterizando un estilo sobreprotector o punitivo. Esto se ve reforzado con el estudio realizado por Sala (2002), quien explica que una mayor puntuación en el estilo sobreprotector se correspondía con menores habilidades intrapersonales; esto lo corrobora Dorta (2012), señalando que las madres con una vaga comprensión de sí mismas, tienden a ser inseguras y temerosas, están al pendiente de sus hijos y vigilan que éstos sigan sus instrucciones sin tratar de entender las emociones de sus hijos, por lo que las madres con alta percepción emocional negativa, se tornaban más distantes afectivamente de sus hijos. En cuanto al estilo asertivo e inhibicionista no se observaron relaciones significativas.

Por otro lado, la relación inversa entre la inteligencia interpersonal y el estilo sobreprotector fue altamente significativa. Este componente de la inteligencia emocional tiene que ver con la empatía, la habilidad para establecer y mantener relaciones mutuamente satisfactorias y con un nivel de responsabilidad social adecuado en el que el propio individuo se siente cooperativo y constructivo de su grupo social. Evidentemente el docente con estilo sobreprotector estaría muy limitado en su capacidad interpersonal. En este sentido, Gallargo (2008) encontró que los docentes con mayor desarrollo de sus habilidades personales y metodológicas desarrollaron mejores actitudes en sus estudiantes, así como una media de calificaciones más altas, de lo cual podría inferirse que el docente con pocas habilidades personales no conseguiría tal influencia social- relacional en sus estudiantes; ya que a menor inteligencia interpersonal mayor presencia de indicadores de un estilo educativo sobreprotector. Así también es posible observar relación inversa y significativa de la inteligencia interpersonal con el estilo educativo punitivo, tal como lo refiere Sala (2002), que el estilo punitivo se corresponde con menores habilidades interpersonales.

En cuanto a la adaptabilidad, se encontró que la relación es también inversa y significativa con los estilos sobreprotector y punitivo, es decir, que cuando se incrementaron los puntajes de los estilos señalados, decreció la adaptabilidad. Evidentemente, a menor desarrollo de la habilidad para la solución de problemas, evaluar la correspondencia entre lo experimentado y la realidad y la capacidad para realizar ajustes adecuados a las emociones y conductas a diferentes situaciones, los docentes muestran una mayor incidencia de un estilo sobreprotector o punitivo. Es así que los docentes con baja capacidad para adaptarse estarían influenciando negativamente la vida de sus estudiantes, ya que como refiere Extremera y Fernández-Berrocal (2004), los profesores transmiten patrones de conducta, convirtiéndose en un modelo de aprendizaje vicario.

La dimensión de inteligencia emocional manejo del estrés, presentó una relación inversa y significativa con el estilo punitivo. Por lo que un docente con estilo educativo punitivo tendría menor capacidad para manejar el estrés. Observando las subdimensiones de cada variable se encuentra un marcado contraste, ya que como define Baron (1997), el manejo del estrés consta de la habilidad para tolerar y soportar situaciones adversas enfrentándolas de manera activa y positiva, así como la capacidad para postergar un impulso. Mientras que Magáz y García (1998) refieren que el estilo punitivo está caracterizado por la intolerancia e incompre nsión. En su investigación en población parental, Dorta (2012) encontró que bajas puntuaciones en el conocimiento de las propias emociones se correlacionó con padres con tendencia al perfeccionismo y trato hostil, padres censuradores y centrados en la conducta negativa de sus hijos. A diferencia de Sala (2002), quien encontró mayor correspondencia del estilo punitivo con menores habilidades interpersonales. En cuanto al estilo sobreprotector se encuentra también una relación inversamente significativa, debido a la incompatibilidad de características, por lo que el estilo educativo sobreprotector tendría un alto nivel de ansiedad lo que predeciría una mala gestión del estrés.

Finalmente se observó también que el estado de ánimo general se relacionó de manera inversa y significativa con el estilo sobreprotector y punitivo. Es decir que la felicidad y el optimismo estarían muy disminuidos en docentes sobreprotector y punitivos, lo cual no les permitiría sentirse satisfechos de su propia vida, ni ver los aspectos positivos a pesar de la adversidad y los sentimientos negativos. Los siguientes ítems refieren "los profesores deben enseñar a sus alumnos que la vida está llena de dificultades con las que hay que luchar", "pienso que mis alumnos no se merecen ningún premio o elogio por comportarse como deben", "lo mejor es que los alumnos aprendan por los daños que sufren en la vida". Al responder afirmativamente se revela la incapacidad para disfrutar de sí mismo y de otros y la falta de expresión de sentimientos positivos de un docente con estilo punitivo. Puntuaciones altas en este componente se corresponderían con un estilo asertivo según refiere Sala (2002).

\section{Conclusiones y recomendaciones}

En el presente estudio se encontró que existe relación significativa entre la inteligencia emocional y los estilos educativos sobreprotector y punitivo de los docentes de instituciones educativas privadas religiosas de Chosica; dicha relación fue inversa, señalando que, a mayor nivel de inteligencia emocional del docente, menor uso de los estilos educativos señalados. Sin embargo, ésta relación no fue significativa con los estilos inhibicionista y asertivo.

Al finalizar este estudio se recomienda ampliar el número de participantes en futuras investigaciones, ya que se tuvo contrastantes resultados con otras investigaciones en cuanto a la relación entre la inteligencia emocional y los estilos educativos, asertivo e inhibicionista. Además, se sugiere analizar la inteligencia emocional de los docentes con otras variables tales como 
estrategias de manejo de aula o estilos de enseñanza.

\section{Referencias}

Barreda, S. (2012). El docente como gestor del clima de aula. Factores a tener en cuenta. (Tesis de grado de maestría, Universidad de Cantabria, España). Recuperado de http://repositorio.unican.es/xmlui/bitstream/handle/10902/1627/Barreda\%20G\%C3\%B3 mez,\%20Mar\%C3\%ADa\%20Soledad.pdf?sequence=1

Bisquerra, R. (2003). Educación emocional y competencias básicas para la vida Revista de Investigación Educativa, 21(1), 07-43. Recuperado de http://revistas.um.es/rie/article/view/99071/94661

Cerrillo, M. (2003). Educar en valores, misión del profesor. Tendencias pedagógicas (8), 59-68. Recuperado de https://dialnet.unirioja.es/servlet/articulo?codigo=1012020

Dorta, P. (2012). Relación entre estilos educativos y variables emocionales de padres dealumnos de educación infantil de un centro de Tacoronte. (Tesis de grado de maestría, Universidad Internacional de la Rioja, Facultad de Educación, España). Recuperado de http://reunir.unir.net/bitstream/handle/123456789/811/2012_05_21_TFM_ESTUDIO_D EL_TRABAJO.pdf?sequence $=1$

Extremera, N. y Fernández-Berrocal, P. (2004). La importancia de desarrollar la inteligencia emocional en el profesorado. (Artículo). Revista Iberoamericana de Educación. Recuperado de http://www.rieoei.org/deloslectores/759Extremera.PDF

Extremera, N., Fernández-Berrocal, P., Durán, A. (2003). Inteligencia emocional y burnout en profesores. (Trabajo de investigación, Universidad de Málaga España). Recuperado de http://emotional.intelligence.uma.es/documentos/PDF5burnout_en_profesores.pdf

Fernández, J. (2005). Matriz de competencias del docente de educación básica. Revista Ibero- Americana, 36(2). Recuperado de http://rieoei.org/investigacion/939Fernandez.PDF

Freitas, A. (2012). Estilos de enseñanza del profesorado de Educación Superior: Estudio Comparativo España - Brasil. (Tesis Doctoral publicada Universidad de Valladolid, España). Recuperado de https://uvadoc.uva.es/bitstream/10324/2041/1/TESIS249- 130213.pdf

Gallargo, B. (2008). Estilos de docencia y evaluación de los profesores universitarios y su influencia sobre los modos de aprender de sus estudiantes. Revista Española de Pedagogía, 56(241), 425-446. Recuperado de http://www.uv.es/gargallo/Estilos.pdf

Huanca, E. (2012). Niveles de inteligencia emocional de docentes de una institución educativa del distrito de Ventanilla-Callao. (Tesis de titulación, Universidad San Ignacio de Loyola, Perú). Recuperado de http://repositorio.usil.edu.pe/bitstream/123456789/1173/1/2012_Huanca_Niveles\%20de $\% 20$ inteligencia\%20emocional\%20de\%20docentes\%20de\%20una\%20instituci\%C3\%B3 n\%20educativa\%20del\%20distrito\%20de\%20Ventanilla-\%20Callao.pdf

López-Goñi, I. y Goñi Zabala, J. (2009). La competencia emocional en los currículos de formación inicial de los docentes. Un estudio comparativo. Revista de Educación, 357(1), 467-489. Recuperado de http://www.mecd.gob.es/dctm/revista-deeducación/articulosre357/re35721.pdf?documentId=0901e72b8127d1f9

Magaz, Á. y García, M. (1998). Perfil de estilos educativos. Manual de referencia. España: ALBOR-COHS.

Sala, J. y Abarca, M. (2002). Las competencias emocionales de los futuros profesores. Revista Electrónica Interuniversitaria de Formación del Profesorado, 5(3), 1-4. Recuperado de http://www.aufop.com/aufop/uploaded_files/articulos/1227713626.pdf

Sala, J. (2002). Ideas previas sobre la docencia y competencias emocionales en estudiantes de ciencias de la educación. Revista Española de Pedagogía, (223), 543 - 548. Recuperado de file://C:/Users/ALESSANDRA/Downloads/223-08\%20(1).pdf

Ugarriza, N. (2001). La evaluación de la inteligencia emocional a través del inventario de BarOn (I-CE) en una muestra de Lima Metropolitana. Red de Revistas Científicas de América Latina y el Caribe, España y Portugal (4), 129-160. Recuperado de http://www.redalyc.org/articulo.oa?id=147118178005

Villán, S. (2013). Estilos de enseñanza y competencia emocional docente en educación infantil. (Trabajo de titulación, Universidad de Valladolid, España). Recuperado de https://uvadoc.uva.es/bitstream/10324/3574/1/TFG-B.347.pdf 